\section{TATRA \\ MOUNTaiNS \\ Mathematical Publications}

DOI: $10.2478 / \mathrm{tmmp}-2014-0004$

Tatra Mt. Math. Publ. 58 (2014), 37-45

\title{
ON DECOMPOSITION OF GENERALIZED CONTINUITY
}

\author{
Sadik Bayhan - Alev Kanibir — Ivan L. Reilly
}

\begin{abstract}
The aim of this paper is to obtain some decompositions of generalized continuity by providing some new variants of generalized continuous functions. Our partial success is an indication of significant differences between the theory of topological spaces and that of generalized topological spaces.
\end{abstract}

\section{Introduction}

In 8], A. C s ás zá r introduced the notions of generalized topological spaces and generalized neighborhood systems. He also introduced generalized continuous functions by using these concepts and studied some of the properties of these functions. Furthermore, his study of generalized open sets in generalized topological spaces [6]-[10] has led to others defining new concepts and deriving new results. After Császár's foundational ideas, several papers have provided new aspects of the theory of generalized continuous functions. The reader may refer (for example) to [4, 5], 17, [18, 20, 24]-28. In this paper, we give decompositions of generalized continuity and of $g$ - $\alpha$-continuity by providing some new types of generalized continuous functions. We adapt the approach for topological continuity using generalized open sets described by R eilly [32.

In the field of Mathematical Psychology, the notion of knowledge space was introduced by Doig non and F a lmagne [11]. These spaces provide a rigorous mathematical foundation for various practical systems of knowledge assessment. Their theory is enunciated in the book [12 by Doig non and Falmagne, where the mathematics is largely combinatorial and stochastic. It turns out that a knowledge space is exactly a strong generalized topological space in the sense

(C) 2014 Mathematical Institute, Slovak Academy of Sciences.

2010 Mathematics Subject Classification: 54A05, 54C05, 54C08.

Keywords: generalized topology, generalized open sets, $g$-continuity, weak $g$-continuity, $g$ - $L C$-continuity, $g$ - $\mathcal{A}$-continuity, $g$-ic continuity, decomposition of continuity, decomposition of generalized continuity, continuity dual. 
of Császár [8]. Knowledge spaces are certainly the most important current example and application of generalized topological spaces, outside of topology.

In the topological category, the morphisms are continuous functions between topological spaces. Over the years, many generalizations of continuous functions have been introduced. Two of the oldest and most significant are nearly continuous functions and quasi-continuous functions. In 1922, B l u m b e r g [3] defined near continuity for real-valued functions on Euclidean space. He used the term densely approached. This concept was generalized later to functions between general topological spaces by $\mathrm{P}$ ták [29] who used the term nearly continuous, and by $\mathrm{Hus}$ in [16] who called this notion almost continuity. Mashhour, Abd El-Monsef and El-Deeb [23] have used the term precontinuity for nearly continuity. The concepts of nearly continuous and nearly open functions are significant in functional analysis, especially in the context of open mapping theorems and closed graph theorems. Even older is the notion of quasi-continuity, which is intimately connected with the question of separate and joint continuity of functions from $\mathbb{R}^{2}$ to $\mathbb{R}$. According to $\mathrm{B}$ a ir e [1], it was $\mathrm{V}$ olt e r r a who observed that if $f: \mathbb{R} \times \mathbb{R} \longrightarrow \mathbb{R}$ is separately continuous, then for each point $(a, b) \in \mathbb{R} \times \mathbb{R}$, for each disc $D$ with center $(a, b)$ and for each $\varepsilon>0$, there is a disc $D_{1}$ contained in $D$ such that

$$
|f(x, y)-f(a, b)|<\varepsilon
$$

for all points $(x, y)$ in $D_{1}$. This property of separately continuous functions was later called quasi-continuity by $\mathrm{Kem}$ p ist y [19]. This property of functions between topological spaces has subsequently appeared under different names in the literature, for example, the term neighborly was used by Bledsoe [2], almost continuous by Frolík [13], and semicontinuous by L e vine [21].

A decomposition of continuity is a pair of properties of functions between topological spaces each of which is weaker than continuity, and which are together equivalent to continuity. One member of the pair is called a continuity dual of the other. In this paper, we shall attempt to obtain "generalized continuity duals", or decompositions of generalized continuity. In particular, we shall try to obtain generalized topological space versions of the two important decompositions of continuity proved by Ganster and Reilly [14], [15]. These papers provided continuity duals of near continuity and of quasi-continuity, respectively.

It turns out, rather surprisingly, that we are able to obtain a generalized topological space analogue for just one of these two fundamental decompositions of continuity. In fact, we show that the decomposition of generalized continuity corresponding to that of continuity, where one continuity dual is quasi-continuity (see [15]) holds. But the other classical decomposition of continuity, where one continuity dual is near continuity (see [14]) has no parallel result for generalized 


\section{ON DECOMPOSITION OF GENERALIZED CONTINUITY}

continuity. We provide a counter-example for the anticipated theorem (conjecture). So, the behaviour of functions between generalized topological spaces differs from the behaviour of functions between topological spaces in a significant fashion.

\section{Preliminaries}

If a non-empty set $X$ is given with the power set $\exp X$, a subset $g_{X}$ of $\exp X$ is said to be a generalized topology (briefly $G T$ ) on $X$ if and only if $\emptyset \in g_{X}$ and an arbitrary union of elements of $g_{X}$ belongs to $g_{X}$ (see [8]). If $X \in g_{X}$, then $g_{X}$ is a strong GTS. If $g_{X}$ is a GT in $X$, the elements of $g_{X}$ are called $g_{X}$-open sets and their complements are called $g_{X}$-closed sets. The generalized interior of a subset $A$ of $X$, denoted by $i_{g_{X}}(A)$, is the union of the collection of all generalized open sets contained in $A$, i.e., the largest $g_{X}$-open set contained in $A$. The generalized closure of a subset $A$ of $X$, denoted by $c_{g_{X}}(A)$, is the intersection of the collection of all generalized closed sets containing $A$, i.e., the smallest $g_{X}$-closed set containing $A$ (see, e.g., [9]). A set $A \subset X$ is $g_{X}$-semiopen [7] if and only if $A \subset c_{g_{X}}\left(i_{g_{X}}(A)\right), g_{X}$-preopen [9] if and only if $A \subset i_{g_{X}}\left(c_{g_{X}}(A)\right), g_{X}-\alpha$-open [9] if and only if $A \subset i_{g_{X}}\left(c_{g_{X}}\left(i_{g_{X}}(A)\right)\right)$. The classes of all $g_{X}$-semiopen sets, all $g_{X}$-preopen sets, all $g_{X}$ - $\alpha$-open sets on $X$ are denoted by $\sigma\left(g_{X}\right), \pi\left(g_{X}\right), \alpha\left(g_{X}\right)$ (briefly $\left.\sigma, \pi, \alpha\right)$, respectively.

Definition 2.1. Let $\left(X, g_{X}\right)$ and $\left(Y, g_{Y}\right)$ be generalized topological spaces. Then, a function $f: X \longrightarrow Y$ is said to be:

(1) $\left(g_{X}, g_{Y}\right)$-continuous [8] if for each $g_{Y}$-open set $U$ in $Y, f^{-1}(U)$ is $g_{X^{-o p e n}}$ in $X$,

(2) $\left(\alpha, g_{Y}\right)$-continuous [28] if for each $g_{Y}$-open set $U$ in $Y, f^{-1}(U)$ is $g_{X}-\alpha$-open in $X$,

(3) $\left(\sigma, g_{Y}\right)$-continuous [28] if for each $g_{Y^{-}}$open set $U$ in $Y, f^{-1}(U)$ is $g_{X^{-}}$semiopen in $X$,

(4) $\left(\pi, g_{Y}\right)$-continuous [28] if for each $g_{Y}$-open set $U$ in $Y, f^{-1}(U)$ is $g_{X}$-preopen in $X$,

(5) weakly $\left(g_{X}, g_{Y}\right)$-continuous 25$]$ if for each $x \in X$ and each $g_{Y}$-open set $V$ containing $f(x)$, there exists a $g_{X}$-open set $U$ containing $x$ such that $f(U) \subset c_{g_{Y}}(V)$.

The terms $g$-continuous, $g$ - $\alpha$-continuous, $g$-semicontinuous, $g$-precontinuous and weakly $g$-continuous are sometimes used for the properties we have denoted by $\left(g_{X}, g_{Y}\right)$-continuous, $\left(\alpha, g_{Y}\right)$-continuous, $\left(\sigma, g_{Y}\right)$-continuous, $\left(\pi, g_{Y}\right)$-continuous and weakly $\left(g_{X}, g_{Y}\right)$-continuous, respectively. We observe that $\left(\sigma, g_{Y}\right)$ -continuity is the generalized version of the classical notion of quasi-continuity 
of functions between topological spaces, while $\left(\pi, g_{Y}\right)$-continuity is the generalized analogue of nearly continuous functions.

We now recall some notions defined in [10].

Let $g_{X}$ be a $G T$ on $X$ and $f: X_{0} \longrightarrow X$ be a function. Then $\left\{f^{-1}(G): G \in g_{X}\right\}$ constitutes the inverse image $g_{X_{0}}=f^{-1}\left(g_{X}\right)$ of $g_{X}$.

Proposition 2.2 ([10]). If $g_{X}$ is a GT on $X$, then $f^{-1}\left(g_{X}\right)$ is a GT on $X_{0}$.

The $g_{X_{0}}$-closed sets are those of the form $f^{-1}(F)$, where $F$ is $g_{X}$-closed. In the particular case when $X_{0} \subset X$ and $f(x)=x$ for $x \in X_{0}$, then $g_{X_{0}}$ is the restriction of $g_{X} \rightarrow X_{0}$. It is denoted by $g_{X_{0}}=\left.g_{X}\right|_{X_{0}}$. In this case $f^{-1}(G)=G \cap X_{0}$ for $G \subset X$. So $G$ is $g_{X_{0}}$-closed if and only if $G=F \cap X_{0}$, where $F$ is $g_{X}$-closed.

Let $\beta \subset \exp Y$ be arbitrary. Then,

Lemma 2.3 ([10]). The family $g_{Y} \subset \exp Y$ composed of $\emptyset$ and all sets $N \subset Y$ of the form $N=\cup_{i \in I} B_{i}$, where $B_{i} \in \beta$ and $I \neq \emptyset$ is arbitrary, is a GT on $Y$.

Hence we say that the base $\beta$ generates the $G T g_{Y}$.

Lemma 2.4 ([10]). Let $g_{X}$ be a $G T$ on $X$ and the $G T g_{Y}$ on $Y$ be generated by the base $\beta$. Then a map $f: X \longrightarrow Y$ is $\left(g_{X}, g_{Y}\right)$-continuous if and only if $f^{-1}(B) \in g_{X}$ for each $B \in \beta$.

\section{Decomposition of generalized continuity}

In [15], Ganster and Reilly introduced the concept of interior-closed subsets ( $i c$ sets) of a topological space. We adapt their definition for generalized topological spaces in Definition 3.1. Note that a set $H$ is $g_{A}$-closed in $A \subset\left(X, g_{X}\right)$ if $H=F \cap A$ for some $g_{X}$-closed subset $F$ of $\left(X, g_{X}\right)$.

Definition 3.1. Let $\left(X, g_{X}\right)$ be a generalized topological space. A set $A \subset X$ is called $g_{X}$-interiorclosed (briefly $g_{X}-i c$ ) if $i_{g_{X}}(A)$ is $g_{A^{-}}$-closed in $A$.

It is clear that any $g_{X}$-open set is $g_{X}$-ic but the converse is not true. A simple example for the case of topological space may be given (see Example 1 of [15]).

Now, Definition 3.1 allows us to obtain a theorem analogous to one of the main theorems of [15]. However, our method of proof is not entirely parallel to the corresponding proof in [15].

TheOREM 3.2. Let $\left(X, g_{X}\right)$ be a generalized topological space and $A \subset X$. Then $A$ is $g_{X}$-open if and only if $A$ is $g_{X}-i c$ and $g_{X}$-semiopen. 
Proof. Sufficiency is obvious. If $A$ is $g_{X}$-ic, then $i_{g_{X}}(A)=F \cap A$, where $F$ is $g_{X}$-closed. Since $c_{g_{X}}\left(i_{g_{X}}(A)\right)$ is the smallest $g_{X}$-closed subset containing $i_{g_{X}}(A)$, we have $i_{g_{X}}(A)=c_{g_{X}}\left(i_{g_{X}}(A)\right) \cap A$. If $A$ is $g_{X^{-}}$semiopen, then $A \subset c_{g_{X}}\left(i_{g_{X}}(A)\right)$, so that $i_{g_{X}}(A)=c_{g_{X}}\left(i_{g_{X}}(A)\right) \cap A \supset A$. Hence, $A$ is $g_{X}$-open.

Again, following the pattern established by Ganster and Reilly [14, we now define a new class of functions between generalized topological spaces.

Examples from [15] can be used to distinguish between functions satisfying Definition 3.3 and functions not having this property.

Definition 3.3. Let $\left(X, g_{X}\right)$ and $\left(Y, g_{Y}\right)$ be generalized topological spaces. Then a function $f: X \longrightarrow Y$ is called $\left(i c, g_{Y}\right)$-continuous (or briefly $g$-icc) if for each $g_{Y}$-open set $U$ in $Y, f^{-1}(U)$ is $g_{X}-i c$ in $X$.

The proof of the following decomposition of $\left(g_{X}, g_{Y}\right)$-continuity follows from Theorem 3.2 and Definitions 3.1, 3.3, and 2.1.

TheOREM 3.4. Let $f:\left(X, g_{X}\right) \longrightarrow\left(Y, g_{Y}\right)$ be a function. Then $f$ is $\left(g_{X}, g_{Y}\right)$ -continuous if and only if $f$ is $\left(i c, g_{Y}\right)$-continuous and $\left(\sigma, g_{Y}\right)$-continuous.

In 1985, Reilly and Vamanamurthy 31 proved for any topological space $(X, \tau)$ that a subset $A$ of $X$ is $\alpha$-open if and only if $A$ is semi-open and preopen. Twenty years later, Csás zár [9] obtained the corresponding result for generalized topologies. It is stated as our next theorem.

Theorem $3.5(9])$. If $g_{X}$ is a GT on $X$, then $\alpha\left(g_{X}\right)=\sigma\left(g_{X}\right) \cap \pi\left(g_{X}\right)$.

We have a proof of the following decomposition for $\left(\alpha, g_{Y}\right)$-continuity from Theorem 3.5 and Definition 2.1.

Theorem 3.6. Let $f:\left(X, g_{X}\right) \longrightarrow\left(Y, g_{Y}\right)$ be a function. Then $f$ is $\left(\alpha, g_{Y}\right)$ -continuous if and only if $f$ is $\left(\sigma, g_{Y}\right)$-continuous and $\left(\pi, g_{Y}\right)$-continuous.

\section{Differences from decomposition of continuity}

There are two important decompositions of continuity between (ordinary) topological spaces. Let $X$ and $Y$ be topological spaces and $f: X \longrightarrow Y$ be a function. Then,

A. $f$ is continuous if and only if $f$ is nearly continuous and $L C$-continuous [14], and

B. $f$ is continuous if and only if $f$ is quasi-continuous and $i c$-continuous [15]. 
In this section we show the surprising result that decomposition $A$ does not have an analogous result for generalized topological spaces, while decomposition B has an exactly parallel decomposition for generalized continuity (namely Theorem 3.5). This dichotomy is unexpected.

Recall that a subset $R$ of the generalized topological space $\left(X, g_{X}\right)$ is defined to be $g_{X}$-regular open (or $g_{X} r$-open) if $R=i_{g_{X}}\left(c_{g_{X}}(R)\right)$. The complements of $g_{X}$-regular open sets are called $g_{X}$-regular closed.

Definition 4.1 ([30]). Let $\left(X, g_{X}\right)$ be a generalized topological space and $A \subset X$. Then, $A$ is said to be:

(1) $g_{X}$-locally closed if $A=U \cap F$, where $U$ is $g_{X}$-open and $F$ is $g_{X}$-closed,

(2) a $g_{X}-\mathcal{A}$-set if $A=U \cap F$, where $U$ is $g_{X}$-open and $F$ is $g_{X}$-regular closed.

We shall denote the collections of $g_{X}$-locally closed and $g_{X}$ - $\mathcal{A}$-sets by $L C\left(g_{X}\right)$ and $\mathcal{A}\left(g_{X}\right)$, respectively. Similarly to the classical case, a subset $A$ of $\left(X, g_{X}\right)$ is $g_{X}$-locally closed if $A=U \cap c_{g_{X}}(A)$ for some $g_{X}$-open set $U$.

In topological spaces, it is the case that every open subset is locally closed and that every closed subset is locally closed, see Ganster and Reilly [14]. The corresponding situation in generalized topological spaces is quite different. In a generalized topological space, every $g_{X}$-open set is $g_{X}$-locally closed but every $g_{X}$-closed set is not $g_{X}$-locally closed in general. Furthermore, every $g_{X}-\mathcal{A}$-set is $g_{X}$-locally closed but the converse is not true.

ExAmple 4.2. Let $X=\{a, b, c, d\}$ and $g_{X}=\{\emptyset,\{b\},\{b, c, d\}\}$. Then $g_{X}$ is a $G T$ on $X$. Furthermore, the $g_{X}$-closed subsets are $\{X,\{a, c, d\},\{a\}\}$ and $L C\left(g_{X}\right)=g_{X} \cup\{\{c, d\}\}$, and $\mathcal{A}\left(g_{X}\right)=g_{X}$. Then the subset $\{a, c, d\}$ is $g_{X}$-closed but it is not $g_{X}$-locally closed. Also $\{c, d\}$ is $g_{X}$-locally closed but it is not a $g_{X}-\mathcal{A}$-set.

Definition 4.3. Let $\left(X, g_{X}\right)$ and $\left(Y, g_{Y}\right)$ be generalized topological spaces. Then a function $f: X \longrightarrow Y$ is said to be:

(1) $\left(L C, g_{Y}\right)$-continuous [22, or $g$ - $L C$-continuous, if for each $g_{Y}$-open set $U$ in $Y, f^{-1}(U)$ is $g_{X}$-locally closed in $X$,

(2) $\left(\mathcal{A}, g_{Y}\right)$-continuous, or $g$ - $\mathcal{A}$-continuous, if for each $g_{Y}$-open set $U$ in $Y$, $f^{-1}(U)$ is a $g_{X}-\mathcal{A}$-set in $X$.

It is clear that $\left(\mathcal{A}, g_{Y}\right)$-continuity implies $\left(L C, g_{Y}\right)$-continuity but the converse relation may not be true in general. A simple example for the case of topological spaces may be given (see [14, Example 1]).

In [14, the following theorem was proved for topological spaces. But we observe from Example 4.5 below that the generalized analogue of Theorem 4.4 does not hold in generalized topological spaces. One reason for that difference is 


\section{ON DECOMPOSITION OF GENERALIZED CONTINUITY}

because in generalized topological spaces we do not necessarily have the preservation of the family of $g_{X}$-open sets under finite intersection.

ThEOREM 4.4 ([14]). Let $f: X \longrightarrow Y$ be a function between topological spaces $X$ and $Y$. Then,

(1) $f$ is continuous if and only if $f$ is precontinuous and $L C$-continuous,

(2) $f$ is continuous if and only if $f$ is precontinuous and $\mathcal{A}$-continuous,

(3) $f$ is continuous if and only if $f$ is $\alpha$-continuous and LC-continuous,

(4) $f$ is $\mathcal{A}$-continuous if and only if $f$ is semicontinuous and $L C$-continuous.

EXAMPLE 4.5. Let $X=\{a, b, c, d, e\}$ and $g_{X}=\{\emptyset,\{a\},\{a, b, c\},\{a, b, d\},\{a, b, c, d\}$, $\{c, e\},\{a, c, e\},\{a, b, c, e\}, X\}$. Then, $g_{X}$ is a $G T$ on $X$. The subset $\{a, b\}$ is $g_{X}-\alpha$-open since $\{a, b\} \subset i_{g_{X}}\left(c_{g_{X}}\left(i_{g_{X}}(\{a, b\})\right)\right)$. Furthermore, $\{a, b\}$ is $g_{X}$-locally closed since $\{a, b\}=\{a, b, c\} \cap\{a, b, d\}$, where $\{a, b, c\}$ is a $g_{X}$-open set and $\{a, b, d\}$ is a $g_{X}$-closed set.

Now, let $Y=X$ and let $g_{Y}=\{\emptyset,\{a, b\}\}$. Then, $g_{Y}$ is a $G T$ on $Y$.

Let $f:\left(X, g_{X}\right) \longrightarrow\left(Y, g_{Y}\right)$ be the identity function defined by $f(x)=x$ for each $x \in X$. Since $f^{-1}(\{a, b\})=\{a, b\} \notin g_{X}, f$ is not $\left(g_{X}, g_{Y}\right)$-continuous. On the other hand, since $\{a, b\}$ is $g_{X}$-locally closed, $g_{X}-\alpha$-open and $g_{X}$-A-set, $f$ is $\left(L C, g_{Y}\right)$-continuous, $\left(\alpha, g_{Y}\right)$-continuous and $\left(\mathcal{A}, g_{Y}\right)$-continuous.

We can extend this example to show that Theorem 4.4 (4) does not hold in general for generalized topological spaces. Let $Z=X$ and define $g_{Z}=\{\emptyset,\{c\}\}$. Then $g_{Z}$ is a $G T$ on $Z$. Let $h:\left(X, g_{X}\right) \longrightarrow\left(Z, g_{Z}\right)$ be the identity function. Now, the $g_{X}-\mathcal{A}$-sets are $g_{X} \cup\{\{a, b\},\{c\}\}$. Consider the set $\{c\}$. It is a $g_{X}$ - $\mathcal{A}$-set but it is not $g_{X}$-semiopen. Hence $h$ is $\left(\mathcal{A}, g_{Z}\right)$-continuous, but $h$ is not $\left(\sigma, g_{Z}\right)$-continuous.

Acknowledgements. The authors gratefully acknowledge financial support for this research by the Council of Higher Education in Turkey (YOK), and hospitality from the School of Mathematics, Statistics and Applied Mathematics of NUI Galway.

\section{REFERENCES}

[1] BAIRE, R.: Sur les fonctions des variables reelles, Ann. Mat. Pura Appl. 3 (1899), 1-122.

[2] BLEDSOE, W.: Neighborly functions, Proc. Amer. Math. Soc. 3 (1952), 114-115.

[3] BLUMBERG, H.: New properties of all real functions, Trans. Amer. Math. Soc. 24 (1922), 113-128.

[4] BORSÍK, J.: Generalized oscillations for generalized continuities, Tatra Mt. Math. Publ. 49 (2011), 119-125.

[5] BORSÍK, J.: Points of generalized continuities, Tatra Mt. Math. Publ. 52 (2012), 153-160. 


\section{SADIK BAYHAN — ALEV KANIBIR — IVAN L. REILLY}

[6] CSÁSZÁR, A.: Generalized open sets, Acta Math. Hungar. 75 (1997), 65-87.

[7] CSÁSZÁR, A.: On the $\gamma$-interior and $\gamma$-closure of a set, Acta Math. Hungar. 80 (1998), 89-93.

[8] CSÁSZÁR, A.: Generalized topology, generalized continuity, Acta Math. Hungar. 96 (2002), 351-357.

[9] CSÁSZÁR, A.: Generalized open sets in generalized topologies, Acta Math. Hungar. 106 (2005), 53-66.

[10] CSÁSZÁR, A.: Normal generalized topologies, Acta Math. Hungar. 115 (2007), 309-313.

[11] DOIGNON, J-P.-FALMAGNE, J-C.: Spaces for the assessment of knowledge, Internat. J. Man-Machine Studies 23 (1985), 175-196.

[12] DOIGnON, J-P.-FALMAGNE, J-C.: Knowledge Spaces. Springer-Verlag, Berlin, 1999.

[13] FROLÍK, Z.: Remarks concerning the invariance of Baire spaces under mappings, Czechoslovak Math. J. 11 (1961), 381-385.

[14] GANSTER, M.-REILly, I. L.: A decomposition of continuity, Acta Math. Hungar. 56 (1990), 299-301.

[15] GAnsteR, M.-REILlY, I. L.: Another decomposition of continuity, Ann. New York Acad. Sci. 704 (1993), 135-141.

[16] HUSAIN, T.: Almost continuous mappings, Prace Mat. 10 (1966), 1-7.

[17] KANIBIR, A.-REILLY, I. L.: Generalized continuity for multifunctions, Acta Math. Hungar. 122 (2009), 283-292.

[18] KANIBIR, A.-REILLY, I. L.: On generalized continuity and openness for set-valued functions, Acta Math. Hungar. 126 (2010), 369-380.

[19] KEMPISTY, S.: Sur les fonctions quasicontinues, Fund. Math. 19 (1932), 184-197.

[20] KORCZAK-KUBIAK, E.-LORANTY, A.-PAWLAK, R. J.: Baire generalized topological spaces, generalized metric spaces and infinite games, Acta Math. Hungar. 140 (2013), 203-231.

[21] LEVINE, N.: Semi-open sets and semi-continuity in topological spaces, Amer. Math. Monthly 70 (1963), 36-41.

[22] MARY, S. D.-NAGAVEnI, N.: A new class of locally closed sets and locally closed continuous functions in generalized topological spaces, Int. J. Comput. Appl. 49 (2012), 28-30.

[23] MASHHOUR, A. ABD.-EL-MONSEF, M.-EL-DEEB, S.: On pre-continuous and weak pre-continuous mappings, Proc. Math. Phys. Soc. Egypt 53 (1982), 47-53.

[24] MIN, W. K.: Some results on generalized topological spaces and generalized systems, Acta Math. Hungar. 108 (2005), 171-181.

[25] MIN, W. K.: Weak continuity on generalized topological spaces, Acta Math. Hungar. 124 (2009), 73-81.

[26] MIN, W. K.: Almost continuity on generalized topological spaces, Acta Math. Hungar. 125 (2009), 121-125.

[27] MIN, W. K.: A note on $\theta\left(g, g^{\prime}\right)$-continuity in generalized topological spaces, Acta Math. Hungar. 125 (2009), 387-393.

[28] MIN, W. K.: Generalized continuous functions defined by generalized open sets on generalized topological spaces, Acta Math. Hungar. 128 (2010), 299-306.

[29] PTÁK, V.: Completeness and open mapping theorem, Bull. Soc. Math. France 86 (1958), $41-74$

[30] RANI, R. J.—JEYANTHI, P.—SIVERAJ, D.: More on $\gamma$-interior, Bull. Allahabad Mat. Soc. 25 (2010), 1-12. 


\section{ON DECOMPOSITION OF GENERALIZED CONTINUITY}

[31] REILLY, I. L.-VAMANAMURTHY, M.: On $\alpha$-continuity in topological spaces, Acta Math. Hungar. 45 (1985), 27-32.

[32] REILLY, I. L.: On generalized open sets and decompositions of continuity, Tatra Mt. Math. Publ. 14 (1998), 213-217.

Received July 17, 2013

Sadik Bayhan

Department of Mathematics

Mehmet Akif Ersoy University

15030, Örtülü, Burdur

TURKEY

E-mail: bayhan@mehmetakif.edu.tr

Alev Kanibir

Department of Mathematics

Hacettepe University

06532 Beytepe, Ankara

TURKEY

E-mail: kanibir@hacettepe.edu.tr

Ivan L. Reilly

Department of Mathematics

University of Auckland

P.B. 92019, Auckland

NEW ZEALAND

E-mail: i.reilly@auckland.ac.nz 